\title{
Phase shifts in multi-frequency observations of the drift bands of J0034-0721
}

\author{
Samuel J. McSweeney ${ }^{1,2}$, N. D. Ramesh Bhat ${ }^{1,2}$, \\ Steven E. Tremblay ${ }^{1,2}$ and Avinash A. Deshpande ${ }^{3}$ \\ ${ }^{1}$ International Centre for Radio Astronomy Research (ICRAR), Curtin University \\ 1 Turner Ave., Technology Park, Bentley, WA 6102, Australia \\ ${ }^{2}$ ARC Centre of Excellence for All-sky Astrophysics (CAASTRO) \\ ${ }^{3}$ Raman Research Institute (RRI) \\ C. V. Raman Avenue, Sadashivanagar, Bengaluru 560 080, India
}

\begin{abstract}
We observed single pulses from PSR J0034-0721 (B0031-07) simultaneously at the MWA $(185 \mathrm{MHz})$ and the GMRT $(610 \mathrm{MHz})$. Correlation analyses reveal that the phase difference of the average profiles at the two frequencies differs from the phase difference observed between individual subpulses, indicating that the individual emission columns above the pulsar's rotating carousel of sparks do not evolve in frequency in the same way that the global magnetosphere does. This hints at a possible departure from the dipolar field geometry in this pulsar's emission region. Moreover, the discrepancy depends on the drift mode, suggestive of a way to constrain the emission heights associated with each drift mode.
\end{abstract}

Keywords. Pulsars: general, pulsars: individual (J0034-0721)

\section{Introduction}

The pulsar phenomenon of sub-pulse drifting exhibits many well-known broadband effects, including nulling, mode switching, and drift-mode switching (Gajjar et al. 2014 and references therein). We have undertaken simultaneous, multi-frequency observations of PSR J0034-0721, a well-known subpulse drifter that has three distinct drift modes, labelled as $\mathrm{A}, \mathrm{B}$, and $\mathrm{C}$, in order from largest to smallest $P_{3}$ values (Huguenin et al., 1970, McSweeney et al., 2017). Previous multi-frequency, simultaneous studies focused on the frequency evolution of the average mode profiles, interpreting the observed difference between the drift modes geometrically as corresponding to different colatitudes of the carousel of sparks with respect to the magnetic pole (Smits et al., 2005, 2007). We obtained low frequency data $(185 \mathrm{MHz})$ with the Murchison Widefield Array (MWA; Tingay et al., 2013), and higher frequency data with the Giant Metrewave Radio Telescope (GMRT). Here, we argue that the frequency evolution of individual pulses (measured in terms of a drift-mode-dependent phase shift) is inconsistent with the frequency evolution of the average mode profiles, if the usual dipolar field geometry is assumed.

\section{Overview}

Under the assumptions of curvature radiation, the particles ultimately responsible for pulsar radio emission are constrained to follow magnetic field lines as they propagate away from the central star (Radhakrishnan \& Cooke, 1969). In the carousel model, only those field lines connected to spark events near the surface are visible at any given moment, implying that a subpulse observed at two frequencies (i.e. at two altitudes) are emitted simultaneously. Therefore, any observed lag between the times of arrival of a single subpulse at two frequencies must be due to the different beaming geometry at the two heights due to the dipolar structure of the field (coupled with an assumed 


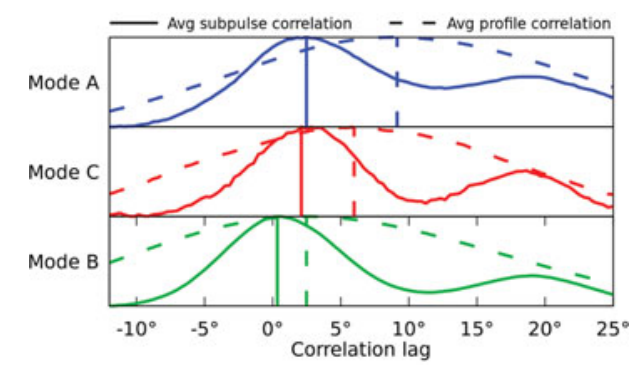

Figure 1. The dual-frequency subpulse correlation lags (solid lines) relative to the dual-frequency average profile correlation lags (dashed) for the three drift modes of PSR J0034-0721. The average profile lags encapsulate "global" effects such as aberration and retardation, which are not entirely accounted for in the subpulse correlation lags.

radius-to-frequency mapping; Cordes, 1978), as well as any aberration and retardation (AR) effects, which are also height-dependent.

Subpulse phase lags caused by AR effects affect the magnetosphere globally, in the sense that the phase lag is observed as an equivalent redistribution of flux in the average profile (which is the usual context for seeing AR effects in action). Thus, even when the absolute time offset between two simultaneous observations (recorded at two telescopes) is not known, one can "normalise out" AR effects by measuring the phase lags of the subpulses with respect to the average profiles. This can be achieved by cross-correlating individual pulses and comparing them (individually, or in bulk by first averaging the cross-correlations together) to the cross-correlation of the average profiles at the two frequencies. Doing this for our MWA and GMRT observations shows that there still remains a significant subpulse lag that is not related to AR effects and which changes depending on which drift mode is present (see Fig 1).

\section{Implications}

In each drift mode, there appears to be a subpulse lag (compared across two frequencies) that cannot be simply explained by AR effects, or, indeed, by an appeal to a simple radius-to-frequency mapping in a dipole geometry (since this too would be "normalised out" by comparison to the average profile). Thus, we conclude that either (1) the active (i.e. visible) field lines are not strictly dipolar in the emission region of this pulsar, or (2) the magnetic field lines are not emitting simultaneously along their length (within the emission region). If the former, we consider the possibility that multipolar components are becoming significant at the relevant heights; if the latter, that the condition for coherent emission may take the form of some kind of plasma wave whose direction of propagation has a radial component. In either case, this interesting and unique pulsar may yet prove an important tool for studying the underlying radio emission mechanism.

\section{References}

Cordes, J. M. 1978, ApJ, 222, 1006-1011

Gajjar, V., Joshi, B. C., Kramer, M., Karuppusamy, R., \& Smits, R. 2014, ApJ, 797, 18

Huguenin, G. R., Taylor, J. H., \& Troland, T. H. 1970, ApJ, 162, 727-735

Maan, Y. \& Deshpande, A. A. 2014, ApJ, 792, 130

McSweeney, S. J., Bhat, N. D. R.., Tremblay, S. E., Deshpande, A. A., \& Ord, S. M. 2017, ApJ, 836,224

Radhakrishnan, V. \& Cooke, D. J. 1969, Ap. Lett., 3, 225

Smits, J. M., Mitra, D., \& Kuijpers, J. 2005, A\&A, 440, 10

Smits, J. M., Mitra, D., Stappers, B. W., Kuijpers, J., Weltevrede, P., \& Jessner, A. Gupta, Y. 2007, A\&SA, 465, 15 